\title{
A Novel Approach to Wideband Spectrum Compressive Sensing Based on DST for Frequency Availability in LEO Mobile Satellite Systems
}

\author{
Feilong Li, Guangxia Li, Zhiqiang Li, Yulin Wang, and Gengxin Zhang \\ Institute of Communication Engineering, PLA University of Science and Technology, Nanjing 210007, China \\ Correspondence should be addressed to Feilong Li; 15895970727@163.com
}

Received 22 February 2016; Accepted 4 July 2016

Academic Editor: Cornel Ioana

Copyright (c) 2016 Feilong Li et al. This is an open access article distributed under the Creative Commons Attribution License, which permits unrestricted use, distribution, and reproduction in any medium, provided the original work is properly cited.

In LEO mobile satellite network, the L/S frequency availability is an essential task for global communication but entails several major technical challenges: high sampling rate required for wideband sensing, limited power and computing resources for processing load, and frequency-selective wireless fading. This paper investigates the issue of frequency availability in LEO mobile satellite system, and a novel wideband spectrum compressed signal detection approach is proposed to obtain active primary users (PUs) subbands and their locations that should be avoided during frequency allocation. We define the novel wideband spectrum compressed sensing method based on discrete sine transform (DST-WSCS), which significantly improves the performance of spectrum detection and recovery accuracy compared with conventional discrete Fourier transform based wideband spectrum compressed sensing scheme (DFT-WSCS). Additionally, with the help of intersatellite links (ISL), the scheme of multiple satellites cooperative sensing according to OR and MAJ decision fusion rules is presented to achieve spatial diversity against wireless fading. Finally, in-depth numerical simulations are performed to demonstrate the performance of the proposed scheme in aspect of signal detection probability, reconstruction precision, processing time, and so forth.

\section{Introduction}

Currently, there is a renewed $\mathrm{R} \& \mathrm{D}$ interest and market opportunities for mobile satellite systems (MSS), which have been utilized for providing communication services to fixed and mobile users for a vast range of sectors (e.g., land-mobile, aeronautical, maritime, transports, rescue and disaster relief, and military) where terrestrial infrastructures are congested or infeasible to be deployed [1]. According to the orbit types, the operational MSS can be categorized into GEO based MSS and non-GEO based MSS. In those systems, the satellite antenna has multiple spot beams that irradiate cells on the earth, thus creating a cellular-like coverage. Each orbit has some desirable properties as well as a set of drawbacks for efficient service delivery. However, it is impossible to be equipped with large antennas and high power supply for mobile terminals that need to communicate with each other around the world, such as handsets; the idea of employing lower altitude satellites is put into practice. Details of the satellite orbit, user link frequency band, physical layer, multiple access, satellite features, and intersatellite links (ISL) can be found in [2-5].

Frequency for MSS is assigned at the World Radiocommunication Conferences (WRC) periodically organized by the International Telecommunication Union-Radiocommunication (ITU-R) sector. While fixed services use high $\mathrm{C}$ and $\mathrm{K}$ frequency band, mobile services are better suited for $\mathrm{L}$ and $\mathrm{S}$ frequency band allocated at the World Administrative Radio Conference (WARC) 92 [6]. The limitation of L/S band resources and the rapid growth demand for broadband data services are a push toward the use of $\mathrm{Ku} / \mathrm{Ka}$ band for MSS. $I T U-R$ has assigned portion of Ka frequency band to MSS and fixed satellite systems (FSS) on a primary basis in all regions (29.9-30 GHz for earth-to-space link and 20.1-21.3 GHz for 
space-to-earth link) and $\mathrm{Ku}$ frequency band to MSS on a secondary basis in all regions $(14-14.5 \mathrm{GHz}$ for earth-tospace link and $10-12 \mathrm{GHz}$ for space-to-earth link). In fact, the systems adopted $\mathrm{Ku} / \mathrm{Ka}$ are mainly intended for fixed users with big antennas for higher signal attenuation and impact of atmospheric effects and cannot provide a good coverage over seas, because antenna spot-beams footprints are focused on landmass [7]. L/S frequency band has been exploited by MSS for a long time due to permission for small on-board antennas and personal communication anywhere with anyone at anytime. In other words, it is obvious that almost all the operational MSS regard L/S band as the preferential frequency resource to support mobile users.

According to the final documentation of WRC held in Geneva, Switzerland, 2012 (WRC-12), the allotment of L/S band for mobile satellite services promulgated by ITU Radio Regulation (ITU-RR) is listed in Table 1. In order to reuse frequency and alleviate interference, ITU-RR divides the earth into three regions: Region I (e.g., Europe, Africa, Soviet Russia situated in Asia, and Asia countries lying in the west of Mongolia and Iran's western borderline), Region II (e.g., Latin America, North America, Greenland, and Hawaii), and Region III (e.g., Oceania and the remaining district of Asia).

The statistical results of L/S band used by operational systems obviously show that it is impossible to find a completely pure L/S subband for another planned global mobile satellite system, which has gained much attention in those countries, for example, China, Russia, and European Union, that have not owned such an independently controllable system. The scarcity of $\mathrm{L} / \mathrm{S}$ frequency resources becomes one of the crucial reasons that some MSS have to be postponed, such as LEO-based Courier panned by Germany, European Union, and Russia and the 48 polar-orbiting satellite constellation system proposed by China. Inevitably, whatever strategy of frequency availability for any mobile satellite system should consider the frequency allotment and region division for mobile satellite service in a global context and avoid interference with the operational MSS.

In general, the space segment satellites from different countries adopt different signal frequency when providing satellite mobile service to ground segment terminal users. The analysis of each L/S subband assigned to the operational MSS implies that it is not absolutely impossible to actualize user link frequency for those forthcoming systems on the basis of discovering the available vacancy spectrum. Concretely, it can be switched to subband $1668-1675 \mathrm{MHz} / 1518-1525 \mathrm{MHz}$ when the satellite moves out of the coverage area of AlphaSat I-XL. Contrarily, in the area of Europe, Africa, and Middle East, it can be changed to subband 1980-2010 MHz/2170$2200 \mathrm{MHz}$. For high latitude area (i.e., outside $\pm 70^{\circ}$ latitude and polar region), it is manipulable to choose subband 1610-1626.5/2483.5-2500 MHz because Globalstar provides communication services in mid-low latitude zone. Obviously, performing signal detecting for identification of active spectrum becomes the first and the key task to obtain frequency autonomously. In this aspect, it is similar to cognitive radio (CR) which has been found as a competitive solution to the issue of underutilization of the allocated spectrum [8]. The purpose of spectrum sensing technology in CR is to discover the vacant spectrum to actualize utilization of the unoccupied spectrum by cognitive radio users, and the objective for application of spectrum sensing in MSS is to seek available frequency to provide mobile communication services.

In cognitive radio networks (CRN), secondary users (SUs) need sense the wideband spectrum fleetly and accurately in order to utilize the unused spectrum, as well as avoiding interference to PUs. The methodology significantly improves the use efficiency of the limited radio frequency resource as a result of a low percentage of the whole wideband spectrum taken by PUs. Recently, wideband spectrum sensing represents an effective solution to the issue of simultaneous sensing of the spectrum occupancy of multiple signals spreading over a wide frequency range [9]. However, in wireless network, including satellite network, current analogto-digital hardware technology faces tremendous challenge posed by conventional spectral estimation method which operates in a way equal to or greater than the Nyquist sampling rate. Besides, time-varying spectrum sensing only collects a small quantity of measurements for detecting, which makes it extremely difficult to fulfill high-resolution signal reconstruction reliably.

With the recent advances in the theory of compressed sensing (CS), signal sparsity has been applied to reduce the number of measurements while maintaining high recovery accuracy $[10,11]$. Compressed sampling (i.e., combined sampling with compression operations) as the efficient method to achieve real-time spectrum estimation at a sub-Nyquist sampling rate has attracted substantial research efforts, which is motivated by the fact that there are plenty of temporarily unemployed spectrum holes in the wideband scenario. The compressed sampling theory points out that any sparse signal can be compressed and sampled simultaneously. Preliminary work has been done to exploit CS for wideband spectrum compressed sensing based upon signal sparsity in frequency domain. [12] firstly carried out the work to take advantage of CS theory to acquire wideband signals using subNyquist sampling rate. Recently, [13] presented a structured compressed sensing. And [14] proposed a power spectrum blind sampling (PSBS) algorithm trying to reconstruct the power spectrum. However, all the aforementioned methods belonged to the category of discrete Fourier transform (DFT) based wide spectrum compressed sensing with complexexponential functions set as orthogonal sparse base, which is not the only orthogonal basis used to reconstruct wideband communication signals. Furthermore, a wideband spectrum estimating scheme based on cyclic spectrum, considering the $2 \mathrm{D}$ sparse signal of interest in the cyclic spectrum domain, was proposed in [15]. With the purpose of optimizing the compressive sensing recovery of image and audio signal, [16] put forward an adaptive tree structured dictionary of orthogonal bases. For cognitive power line communication (PLC) systems, [17] applied several time-frequency transforms included DFT, discrete cosine transform (DCT), and discrete sine transform (DST) to spectrum sensing. Additionally, the cooperative sensing technique has attracted considerable research attention in wideband cognitive radio networks as it is capable of significantly improving the performance of wireless communication systems [18-22]. 
TABLE 1: L/S band assignment for MSS.

\begin{tabular}{|c|c|c|c|c|c|}
\hline L/S band & $\begin{array}{c}\text { Uplink frequency } \\
(\mathrm{MHz})\end{array}$ & $\begin{array}{c}\text { Downlink frequency } \\
(\mathrm{MHz})\end{array}$ & $\begin{array}{c}\text { Bandwidth } \\
(\mathrm{MHz})\end{array}$ & Area compartmentalized & Operational systems \\
\hline \multirow{3}{*}{ L band } & $1610.0-1626.5$ & $2483.5-2500.0$ & 16.5 & Global & Iridium, Globalstar \\
\hline & $1626.5-1660.5$ & $1525.0-1559.0$ & 34.0 & Global & $\begin{array}{c}\text { Inmarsat, MSAT, } \\
\text { SkyTerra, ACeS, Thuraya }\end{array}$ \\
\hline & $1668.0-1675.0$ & $1518.0-1525.0$ & 7.0 & Global & AlphaSat I-XL \\
\hline \multirow{3}{*}{$S$ band } & $1980-2010$ & $2170-2200$ & 30 & Global & TerreStar, ICO \\
\hline & $2670-2690$ & $2500-2520$ & 20 & Region III & Insat, N-STAR \\
\hline & $2655-2670$ & $2520-2535$ & 15 & Region III & N-STAR \\
\hline
\end{tabular}

To the best of our knowledge, these works are mostly concerning $\mathrm{CR}$ in terrestrial wireless network. However, there have been few corresponding works about LEO-based mobile satellite communication network in the literature up to now. The time-variant environment and limited processing capability in MSS make it markedly different from terrestrial wireless network. The scheme of wideband spectrum compressed sensing (WSCS) not only implies lower computation complexity and shorter processing time but also means lower sampling rate and more accurate reconstruction. Although an enormous amount of research has focused on wideband spectrum sensing in CRN, there still exit the following challenges to provide reliable spectrum detecting in MSS. Firstly, the whole spectrum of L/S band for MSS is extremely wide, which may cause sensing problems of immense computational costs and long processing delay; secondly, the acquirable spectrum in MSS is time-varying because of high speed motion of the satellite; finally, the channel in MSS is typical fading and shadowing channel due to variable space circumstances. Herein, we propose a brand new approach to resolve these problems: the novel wideband spectrum compressed sensing method based on DST, briefly referred to as DST-WSCS, with a set of sinusoidal functions as an orthogonal sparse basis, to provide more accurate recovery and lower processing complexity. Essentially, the superiority of the novel algorithm is attributed to the following two aspects. On the one hand, the representation of signal in DST domain is much sparser than that of DFT domain. On the other hand, the DST uses only real arithmetic, as opposed to the complex-valued DFT. Finally, we also present collaborative compressed sensing architecture to deal with channel fading in the scenario where satellite may be incapable of detecting active signals because there are no means to distinguish white space and fading effect apart.

The rest of this paper is organized as follows. Section 2 describes the system model. Then, Section 3 proposes novel wide spectrum compressed sensing scheme based on DST. Immediately following, a collaborative signal sensing scheme is developed in Section 4. Extensive simulation results are given in Section 5. Finally, conclusions are drawn in Section 6.

\section{System Model Descriptions}

2.1. System Architecture and Signal Model. The system architecture of WSCS for LEO-based MSS is established in this section. As depicted in Figure 1, we consider a CR network

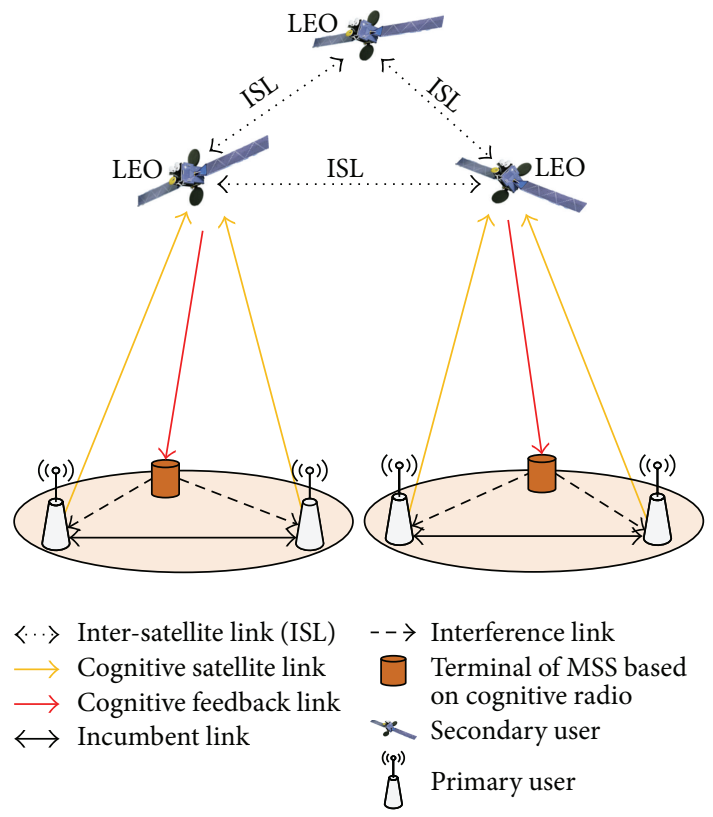

FIGURE 1: System architecture of implementation of compressed sensing for LEO-based MSS.

composed of space segment LEO satellite and terrestrial segment PU, in which LEO satellite is taken as secondary system, while the primary system consisted of PU licensed in the incumbent mobile satellite services. There are broadly three steps to seek available frequency, first in which individual LEO satellite implements compressed sensing to make local decision on the state of PU via cognitive satellite link based on energy detection, second in which LEO choose vacant frequency to communicate with terminal according to sensing decision, and third in which it is selective to actualize cooperative sensing with the help of ISL in the overlapped coverage.

Suppose that the received signal $r(t)$ at SU is spanning over a wide spectrum within $\left(f_{d}, f_{u}\right)$ depicted in Figure 2 , as well as the periodic spectrum sensing time framework, where $0 \sim T_{0}$ is used for implementing compressed spectrum sensing and $T_{0} \sim T$ is reserved for transmitting data.

In particular, the basic assumptions are given as follows:

(i) A slotted frequency segmentation structure is utilized, in which the entire wideband spectrum is 


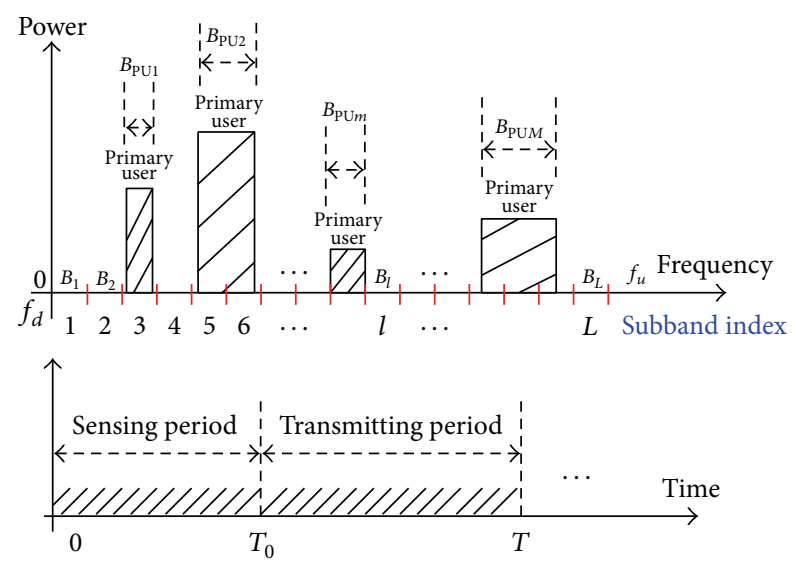

Figure 2: Wideband signal model.

equivalently divided into $L$ orthogonal subbands (also known as slots) centered at $\left\{f_{l}\right\}_{l=1}^{L}$, and the subband index is denoted by $l \in[1, L]$, each of which has bandwidth $B_{l}=(W / L)(\forall l \in[1, L])$. The spectrum set $\left\{B_{\mathrm{PU} 1}, B_{\mathrm{PU} 2}, \ldots, B_{\mathrm{PUm}}, \ldots, B_{\mathrm{PUM}}\right\}$ occupied by potentially active PUs lied within $\left(f_{d}, f_{u}\right)$ consecutively, whose frequency locations and power spectral density (PSD) levels are to be detected and identified. Emphatically, only the unoccupied subbands which accord with the frequency allocated to MSS listed in Table 1 are available.

(ii) It is assumed that signal PSD over each spectrum band $B_{\mathrm{PUm}}$ is smooth and exhibited independently from its two neighboring bands $B_{\mathrm{PU}(m-1)}$ and $B_{\mathrm{PU}(m+1)}$. However, the PSD levels of PUs are dynamically varying, depending on whether they are active or not in a particular time and geographical area. Those temporarily idle subbands are termed spectral holes and available for opportunistic spectrum access by SUs.

(iii) Neither the number of occupied subbands $I(I \leq M)$ nor their locations are known to SUs. For simplicity, let $\Omega_{l m}$ denote the set of subband indices overlapped by $\left\{B_{\mathrm{PUm}}\right\}_{m=1}^{m \leq M}$ in the entire bandwidth, and $\Omega_{l m}=$ $\left\{l \mid l\right.$ represents the subband indices of $B_{\mathrm{PUm}}$ projection in $B_{l}$ \}. Obviously, the active PUs may present at any subband in $\Omega_{l m}$.

(iv) In the spectrum sensing period, all SUs keep quiet as enforced by protocols, for example, at the media access control layer, so that only PUs are emitting power. Meanwhile, the locations and the number of active subbands remain constant but may vary among different sensing period.

Thus, the continuous signal received at the RF frontend of SUs $r(t)$ is composed of only active PUs signals and background noise. Suppose that there are $I$ active PUs during the sensing interval, whose transmitted signals are expressed by $s_{i}(t), i=1, \ldots, I$. We define the channel impulse response between the $i$ th PU transmitter and the $j$ th SU receiver as $h_{i j}(t)$. Thus, the signal $s_{i}(t)$ is reached at the $j$ th satellite detector in the form of the convolution operator $h_{i j}(t) *$ $s_{i}(t)$. We assume that the channel gain can be varying slowly and flat similar to the time-invariant slotted narrowband subchannel during each detection time window. The received signal at the $j$ th detector is given as follows:

$$
r_{j}(t)=\sum_{i=1}^{I} h_{i j}(t) * s_{i}(t)+w_{j}(t),
$$

where the environmental noise around the detector $w_{j}(t)$ is white Gaussian with zero mean and variance $\sigma$. For the time window as $t \in\left[0, T_{0}\right], r_{j}(t)$ have discrete form as an $N$ length signal $r_{t}^{j}$ by using sampling rate $f_{s}$ over the observation time. According to the Nyquist sampling theory, the sampling rate is required at or above $2\left(f_{u}-f_{d}\right)$ samples per second. However, it leads to excessive memory requirement and prohibitive energy cost for wideband spectrum sensing. This dilemma motivates us to employ CS to reduce sampling rate. To represent the discrete signal response on the $I$ channels, we collect the frequency-domain samples into $N \times 1$ vector, and writing every term in discrete version, we have

$$
r_{f}^{(j)}=\sum_{i=1}^{I} h_{f}^{(i j)} s_{f}^{(i)}+w_{f}^{(j)}
$$

where $r_{f}^{(j)}, h_{f}^{(i j)}, s_{f}^{(i)}$, and $w_{f}^{(j)}$ are the complex-valued frequency-domain discrete versions of $r_{j}(t), h_{i j}(t), s_{i}(t)$, and $w_{j}(t)$, respectively. Given (2), the spectrum sensing aims at estimating the transmitted spectrum $\sum_{i=1}^{I} s_{f}^{(i)}$ from PUs, which depends on the a priori knowledge of channel state information (CSI). Identifying spectrum holes is a more important task for SUs, rather than the signal spectrum itself. It turns out to be a spectrum detection problem, aiming at determining the binary spectrum occupancy state $d_{m}(\forall m \in[1, M])$ of active PUs, one-one correspondence with $\left\{B_{\mathrm{PUm}}\right\}_{m=1}^{m \leq M}$. The spectrum detection problem can be solved whether CSI has been acquired or not, because $\sum_{i=1}^{I} s_{f}^{(i)}$ shares the same nonzero support as $\bar{r}_{f}^{(j)}=\sum_{i=1}^{I} h_{f}^{(i j)} s_{f}^{(i)}$, provided that the channel does not experience deep fading and $\sum_{i=1}^{I} h_{f}^{(i j)}$ does not have zero elements. In this case, (2) can be concisely written as

$$
r_{f}^{(j)}=\bar{r}_{f}^{(j)}+w_{f}^{(j)}
$$

As a result of the aforementioned property, we can use the same sampling and reconstruction method to recover either the transmitted $\sum_{i=1}^{I} s_{f}^{(i)}$ for spectrum estimation or the received $\bar{r}_{f}^{(j)}$ for detection of the spectrum support. Besides, we develop collaborative compressive sensing scheme to overcome the drawbacks arising from severe fading and shadowing corrupted in signal with noise in Section 4 later.

2.2. Compressive Sampling. Due to low percentage of spectral occupancy by active PUs, it is believed that the received signal 
$r_{t}^{j}$ at $j$ th SU is sparse in the Fourier domain. As the motivation of this paper, the received signal can be sparsely represented in the frequency domain as follows:

$$
r_{f}^{(j)}=F r_{t}^{j}
$$

where the $N \times 1$ vector $r_{f}^{(j)}$ is the sparse representation of $r_{t}^{j}$ in frequency domain and has just $M \ll N$ nonzero elements. $F$ is an $N \times N D F T$ matrix.

The CS theory states that $r_{t}^{j}$ can be accurately reconstructed by using a sub-Nyquist sampling rate; that is, $f_{s}<$ $2\left(f_{u}-f_{d}\right)$. Mathematically, the compressed samples can be written as

$$
y_{t}^{j}=\Phi r_{t}^{j}
$$

where $\Phi$ is $K \times N$ compressive measurement matrix, columns of $\Phi$ can be viewed as a set of basis functions or matched filters, and different $\Phi$ corresponds to different kinds of sampling. When $\Phi$ is the $N \times N$ identity matrix, model (5) means Nyquist uniform sampling. The $K \times 1$ measurement vector $y_{t}^{j}$ is the projection of $r_{t}^{j}$ onto $\Phi$. And the compression rate is defined $K / N$. Noting that $r_{t}^{j}=F^{-1} r_{f}^{(j)}, F^{-1}$ is inverse of $F$; inserting it into (5), we can get

$$
y_{t}^{j}=\Phi F^{-1} r_{f}^{(j)}=\Phi F^{-1} \bar{r}_{f}^{(j)}+\widetilde{w}_{f}^{(j)},
$$

where $\widetilde{w}_{f}^{(j)}=\Phi F^{-1} w_{f}^{(j)}$ is the AWGN noise vector. As long as $\Phi F^{-1}$ obeys the rule of restricted isometry property (RIP) or the sensing matrix $\Phi F^{-1}$ is incoherent with $F^{-1}$, the occupancy of wideband spectrum could be converted to the reconstruction of $\bar{r}_{f}^{(j)}$, which can be solved by a Min-l1 linear convex optimization with equality constraints in the absence of CSI, as the model given:

$$
\begin{aligned}
\hat{r}_{f}^{(j)}=\arg \min _{\bar{r}_{f}^{(j)}} & \left\|\bar{r}_{f}^{(j)}\right\|_{1} \\
\text { s.t. } \quad & y_{t}^{j}=\left(\Phi F^{-1}\right) \bar{r}_{f}^{(j)} .
\end{aligned}
$$

In order to deal with the noisy signals, as (1) shows, LASSO algorithm has utilized some variants by minimizing the usual sum of squared errors with a bound on the sum of the absolute values of the coefficients. Thus, LASSO has additional advantage of denoising performance. To get the spectrum reconstruction more accurately, we formulate the model based on LASSO as follows:

$$
\begin{aligned}
\hat{r}_{f}^{(j)}=\arg \min _{\bar{r}_{f}^{(j)}} & \left\|\bar{r}_{f}^{(j)}\right\|_{1} \\
\text { s.t. } & \left\|\left(\Phi F^{-1}\right) \bar{r}_{f}^{(j)}-y_{t}^{j}\right\|_{2}^{2}<\varepsilon,
\end{aligned}
$$

where $\varepsilon$ bounds the amount of noise in signals. Great many convex optimization software packages have been developed to tackle the LASSO problem, for example, [23].
2.3. Decision-Making Criterion. After $\bar{r}_{f}^{(j)}$ reconstruction, it is indispensable to estimate the average amplitude of $\widehat{r}_{f}^{(j)}$ within each identified subband for spectrum hole detection. Spectrum detection in local satellite detector can be obtained by a binary hypothesis test problem. We make decision in the lth subband by

$$
d_{l}= \begin{cases}1, & \text { subband } B_{l} \text { is occupied } \\ 0, & \text { subband } B_{l} \text { is unoccupied }\end{cases}
$$

The summary statistic of estimated signal energy over the interval of each subband is computed as the following form:

$$
E_{1}=\sum_{f_{l}-B_{l} / 2}^{f_{l}+B_{l} / 2} \widehat{r}_{f}^{(j)}(f)^{2}
$$

and $\hat{r}_{f}^{(j)}$ is the estimated power spectrum, the decision criterion of $d_{l}=1$ or $d_{l}=0$ is given by

$$
d_{1}= \begin{cases}1, \quad E_{l} \geq \rho \frac{\sum_{l=1}^{L} E_{l}}{L}=\rho \frac{\sum_{l=1}^{L} \sum_{f_{l}-B_{l} / 2}^{f_{l}+B_{l} / 2} \widehat{r}_{f}^{j}(f)^{2}}{L} \\ 0, \quad E_{l}<\rho \frac{\sum_{l=1}^{L} E_{l}}{L}=\rho \frac{\sum_{l=1}^{L} \sum_{f_{l}-B_{l} / 2}^{f_{l}+B_{l} / 2} \widehat{r}_{f}^{j}(f)^{2}}{L},\end{cases}
$$

where $\rho$ is an invariable threshold factor chosen bigger than one. For the whole band, we could get a decision vector $\widehat{\mathbf{d}}^{(j)}=$ $\left(d_{1}^{(j)}, d_{2}^{(j)}, \ldots, d_{l}^{(j)}, \ldots, d_{L}^{(j)}\right)$ over $L$ subbands made by the $j$ th satellite detector that is played by SU depicted in Figure 1. Suppose that there are $J$ detectors simultaneously sensing network wide. Then, we obtain the decision over the $m$ th spectrum bandwidth $B_{\mathrm{PUm}}$ of $d_{m}^{(j)}=1$ or $d_{m}^{(j)}=0$ following the principle

$$
\begin{aligned}
& d_{m}^{(j)} \\
& = \begin{cases}1, & \exists l \in \Omega_{l m} \wedge d_{l}^{(j)}=1, \text { subband } B_{\mathrm{PUm}} \text { is occupied } \\
0, & \text { others, subband } B_{\mathrm{PUm}} \text { is unoccupied. }\end{cases}
\end{aligned}
$$

We could make decision vector $\widehat{\mathbf{d}}^{\prime(j)}=\left(d_{1}^{(j)}, d_{2}^{(j)}, \ldots, d_{m}^{(j)}\right.$, $\ldots, d_{M}^{(j)}$ ) over $M$ spectrum bandwidth in the $j$ th detector. Particularly, in the case of deep fading or shadowing channel, inaccurate detection could be induced when PUs are actually present, which is weighed with detection probability $P_{D}$. And in another case of strong noise, false alarm probability $P_{F}$ may be produced. Thus, $P_{D}$ should be kept as high as possible, whereas the smaller $P_{F}$ will be the better.

\section{A Novel Scheme of Compressed Sensing Based on DST}

For dynamic spectrum access in mobile satellite network, we propose novel algorithm of DST-based compressed spectrum sensing to fulfill better performance in detection probability, reconstruction accuracy, and processing time. 
The DST sequence of $N$-length discrete signal $x(n)$ is defined by

$$
\begin{aligned}
& D(k)=\sum_{n=0}^{N-1} x(n) \sin \left[\frac{\pi}{N}\left(n+\frac{1}{2}\right) k\right] \\
& k=0,1, \ldots, N-1 .
\end{aligned}
$$

Suppose $s(n)$ is a $2 N$-point subtractive symmetry continuation of $x(n)$ represented by

$$
s(n)= \begin{cases}x(n), & 0 \leq n \leq N-1 \\ -x(2 N-n-1), & N \leq n \leq 2 N-1 .\end{cases}
$$

Obviously, the $2 N$-point DFT of $s(n)$ is given by

$$
S(k)=\sum_{n=0}^{2 N-1} s(n) e^{-j(2 \pi n k / 2 N)}
$$

Substituting (14) into (15) yields

$$
\begin{array}{r}
S(k)=\sum_{n=0}^{N-1} x(n) W_{N}^{k n}-\sum_{n=N}^{2 N-1} x(2 N-n-1) W_{N}^{k n}, \\
k=0,1, \ldots, 2 N-1,
\end{array}
$$

where, by definition, $W_{N}^{k n}=\left(e^{-j \pi / N}\right)^{n k}$. If we change the second index of summation using $m=2 N-1-n$ and recall that $W_{N}^{m N}=1$ for integer $m$, then, $S(k)$ could be expressed as

$$
\begin{array}{r}
S(k)=W_{N}^{-k / 2}(-2 j) \sum_{n=0}^{N-1} x(n) \sin \left[\frac{\pi}{N}\left(n+\frac{1}{2}\right) k\right], \\
k=0,1, \ldots, 2 N-1 .
\end{array}
$$

Finally, we obtain the relationship between $S(k)$ and $D(k)$ :

$$
\begin{array}{r}
D(k)=\frac{j}{2} W_{N}^{k / 2} S(k)=\operatorname{Im}\left[-W_{N}^{k / 2} \sum_{n=0}^{N-1} x(n) W_{N}^{k n}\right], \\
k=0,1, \ldots, N-1 .
\end{array}
$$

Im[ ] implies the imaginary part of the term enclosed. DST of $x(n)$ can be computed by taking the $2 N$-point DFT of $s(n)$ and multiplying the result by $(j / 2) W_{N}^{k / 2}$, as shown in (18). We note that $D(k)$ is real, whereas $S(k)$ is complex. Theoretical derivation indicates that the DST basis has better spectral compaction and energy concentration than DFT, which means signal representation is much sparser in DST domain than that of DFT as shown in Figure 3.

On the basis of preceding analysis, the mathematical description of the scheme of DST-WSCS is similar to DFTWSCS scheme with Fourier matrix being replaced by the DST matrix, which can be solved via following three steps:

(i) Estimating sparse sequence $\widehat{r}_{d}^{(j)}$ through compressed measurements $y_{t}^{j}$ at individual $j$ th satellite detector: from (4)-(6), the compressed samples can be represented as

$$
y_{t}^{j}=\Phi r_{t}^{j}=\Phi D^{-1} r_{d}^{(j)}=\Phi D^{-1}\left(\bar{r}_{d}^{(j)}+w_{d}^{(j)}\right),
$$

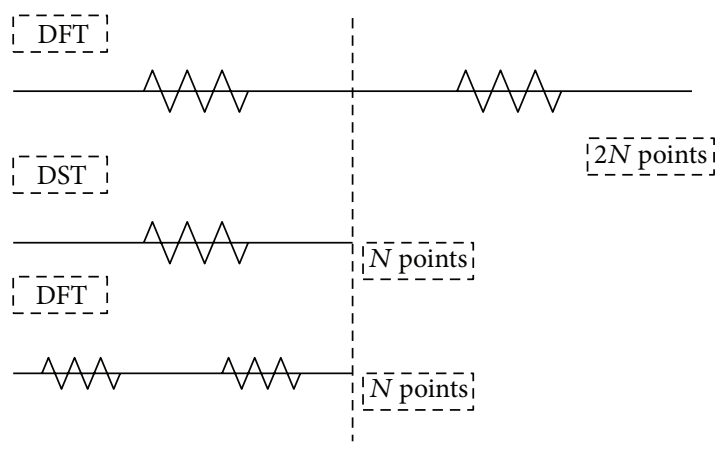

FIGURE 3: Comparison of sparsity between DST and DFT.

where $r_{d}^{(j)}=D r_{t}^{j}$ decomposes into $\bar{r}_{d}^{(j)}$ and $w_{d}^{(j)}$ similar to (3) and $D$ is the $N \times N$ DST matrix. With the same principle as (8), we use LASSO algorithm to get $\widehat{r}_{d}^{(j)}$ from

$$
\begin{aligned}
\widehat{r}_{d}^{(j)}=\arg \min _{\bar{r}_{d}^{(j)}} & \left\|\bar{r}_{d}^{(j)}\right\|_{1} \\
\text { s.t. } & \left\|\left(\Phi F^{-1}\right) \bar{r}_{d}^{(j)}-y\right\|_{2}<\varepsilon .
\end{aligned}
$$

(ii) Reconstructing signal $\widehat{r}_{t}^{j}$ according to $\widehat{r}_{d}^{(j)}$ : as soon as the estimation of DST domain signal $\widehat{r}_{d}^{(j)}$ is accomplished, we could simply get time domain estimated signal, which can be done by the DST inverse transform as

$$
\widehat{r}_{t}^{j}=D^{-1} \widehat{r}_{d}^{(j)}
$$

(iii) Getting frequency response $\widehat{r}_{f}^{(j)}$ from $\widehat{r}_{t}^{j}$ via Fast Fourier Transform (FFT) to identify the state of spectrum usage: We get the frequency estimation given by

$$
\widehat{r}_{f}^{(j)}=\operatorname{FFT}\left(\widehat{r}_{t}^{j}\right) .
$$

Finally, we can get the state of subbands occupied in the whole sensing spectrum according to decision-making rules (10)-(12).

\section{Collaborative Compressed Sensing Scheme}

The wireless channel in MSS is typical fading channel. To obtain reliable detection of spectrum utilization, it is urgent to overcome detrimental effects of the fading. Taking Iridium and 48 polar-orbit constellation for example, they are abundant in satellite resources and intersatellite link (ISL). The probabilities of multiple-satellite coverage is very high; that is, global single-satellite coverage (SSC) rate is up to $100 \%$, double-satellite coverage (DSC) rate is more than $58 \%$, and triple-satellite coverage (TSC) rate is more than $9 \%$, which improve proportionally as the latitude increases, illustrated in Figure 4. All those provide the key factor for the application of cooperative sensing via the user link (UL) and ISL.

The scenario depicted in Figure 5 demonstrates that the spectrum occupancy in the area of DSC and TSC can be cooperatively detected by several satellites located in the same 


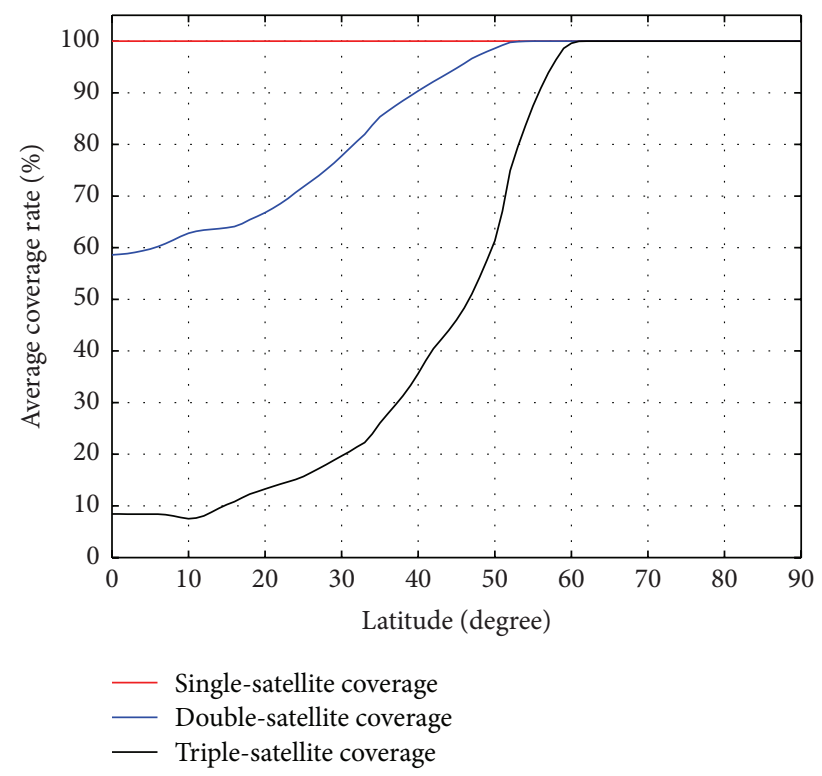

FIGURE 4: The average coverage of 48 polar-orbit constellation.

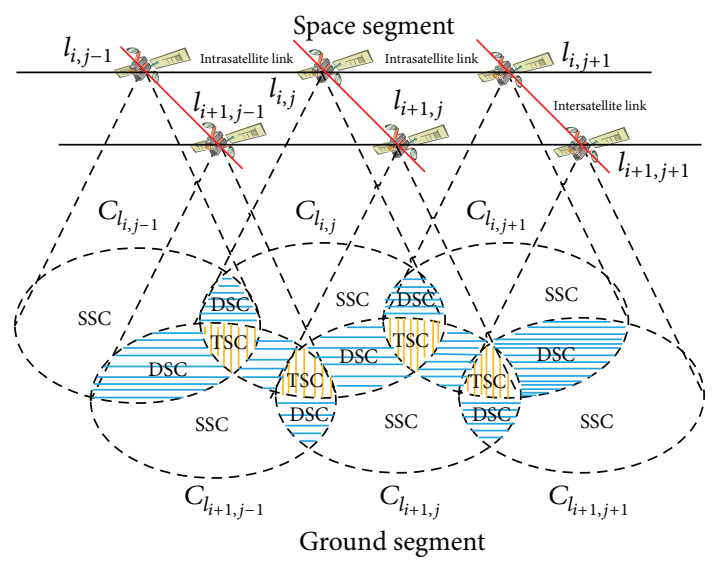

Figure 5: The scenario of collaborative spectrum detection in mobile satellite network.

or adjacent orbit planes, where $C_{l_{i, j}}$ means the coverage area of satellite detector $l_{i, j}$ and the black solid line and red solid line stand for intrasatellite link and intersatellite link, respectively. Plenty of work has shown that collaborative compressive spectrum sensing which exploits the built-in spatial diversity stands out as an effective approach to alleviate the problem of corrupted detection and the sensing burden, as well as cost of individual SU in fading channels.

In distributed mobile satellite network, detector $l_{i, j}$ implements compressive sampling individually to collect reducedrate measurements and possibly make local estimation and decision on the spectrum occupancy of the network. When it comes to data fusion, there are two primary implementation options: centralized and decentralized fusion. However, centralized cooperation scheme requires a fusion center that collects compressed samples or their local decisions from all satellite detectors and makes global sensing decision, which incurs high communication cost and renders the entire network vulnerable to satellite node failure. In this paper, we develop a decentralized cooperative sensing approach to effectively address the abovementioned issue, which relies on the local measurement $\left\{y_{t}^{j}\right\}$, the local sensing estimation $\left\{\bar{r}_{f}^{(j)}\right\}$, and detection decision $\left\{d_{m}^{(j)}\right\}$. The abovementioned collaborative spectrum sensing fusion approach can be, respectively, viewed as measurement fusion, estimation fusion, and decision fusion. A short assessment of their differences would be that measurement fusion needs higher communication overhead compared with the other two, while estimation fusion cannot make $l_{i, j}$ recover the transmitted spectrum $\sum_{i=1}^{I} s_{f}^{(i)}$ individually for the consent on their estimates in the absence of CSI. After weighing the pros and cons, we turn to decision fusion to directly recover the spectrum occupancy vector $d_{m}^{(j)}$ of PUs, which is a binary version with the same nonzero support of $\sum_{i=1}^{I} s_{f}^{(i)}$. To further alleviate communication overhead in terms of processing time and power consumption, we only use local communication among onehop neighbors for cooperation. Let $G(V, E)$ be undirected connected graph depicting the connectivity of the multihop mobile satellite networks, where satellite nodes form the set of vertices $V=\left\{v_{1}, \ldots, v_{i}, \ldots, v_{|V|}\right\}$, where $|V|$ represents the number of satellites in space segment, and each edge $\left(v_{i}, v_{j}\right) \in E, \forall v_{i}, v_{j} \in V$ and $v_{i} \neq v_{j}$, connects an unordered pair of distinct nodes within one-hop neighborhood, which consisted of intra-ISL and inter-ISL. Let $N^{(i, j)}$ denotes the set of one-hop neighbors of satellite detector $l_{i, j}$. We define the channel of UL between active PUs in the ground segment and satellite detector $l_{i, j}$ as detecting channel, as well as reporting channel from the individual detector $l_{i, j}$ to the satellite fusion node that is chosen from $N^{(i, j)}$ sharing the overlapping area with $l_{i, j}$.

The procedure of collaborative compressed sensing follows the steps outlined as follows:

(i) In the processing of detecting channel, each satellite detector $l_{i, j}$ performs local reconstruction to attain $\widehat{r}_{f}^{(j)}$ from compressed measurement $y_{t}^{j}$ via (8) independently and then makes a binary decision vector $d_{m}^{(j)}$ from the nonzero support of $\widehat{r}_{f}^{(j)}$ by (12) to decide whether the spectrum bandwidth $\left\{B_{\mathrm{PUm}}\right\}_{m=1}^{m \leq M}$ is employed or not.

(ii) In terms of reporting channel, each satellite detector $l_{i, j}$ only communicates with its one-hop neighbors $N^{(i, j)}$ to reduce the transmission power consumed during sensing, and the exchanged decision $\left\{d_{m}^{(j)}\right\}$ information percolates over the mobile satellite networks through multiple rounds to make global decision on the spectrum occupancy.

(iii) Assume there are $J$ satellite detectors simultaneously sensing in the mobile satellite network for a particular area, such as the region of SSC, DSC, and TSC. For the utilization state of the whole band, the chosen 
center satellite fuses the local decisions and makes final decision vector $\widehat{d}_{m}$ by taking OR logic rule, as (22) shows,

$$
\widehat{d}_{m}=\operatorname{OR}\left(d_{m}^{(1)}, d_{m}^{(2)}, \ldots, d_{m}^{(J)}\right),
$$

or taking majority vote from the local binary decisions on $d_{m}^{(j)}$, termed as MAJ rule, as (23) shows,

$$
\widehat{d}_{m}= \begin{cases}1, & \sum_{j=1}^{J} d_{m}^{(j)} \geq\left\lfloor\frac{J}{2}\right\rfloor, \\ 0, & \sum_{j=1}^{J} d_{m}^{(j)}<\left\lfloor\frac{J}{2}\right\rfloor,\end{cases}
$$

where the symbol $\lfloor J / 2\rfloor$ denotes the operation of taking the minimum integer that is greater than or equal to $J / 2$. Finally, we could make a decision vector $\widehat{\mathbf{d}}^{\prime}=$ $\left(\widehat{d}_{1}, \widehat{d}_{2}, \ldots, \widehat{d}_{m}, \ldots, \widehat{d}_{M}\right)$ over $M$ spectrum bandwidth for the whole band detection.

\section{Simulations}

This section provides extensive computer simulations to validate the performance of the proposed wideband spectrum compressed sensing scheme and evaluate the effects of key design parameters and network operating condition. Firstly, we describe the simulation setup and relevant performance metrics. Then, for the scenario without cooperation, we compare steady-state performance of proposed novel scheme DST-WSCS with conventional DFT-WSCS and investigate the running time, as well as the impact of system parameters such as SNR, the number of active PUs, and the compression ratio. Finally, for collaborative compressed sensing scenario, we also research the effects of number of cooperators and decision fusion rules on the sensing performance under different values of SNR.

5.1. Simulation Setup and Performance Metrics. As shown in Table 1, the uplink frequency allotted to incumbent MSS located at $1610.0-1660.5 \mathrm{MHz}, 1668.0-1675.0 \mathrm{MHz}$, 1980-2010 MHz, and 2655-2690 MHz. Here, we consider signal with frequency randomly distributed between $1610 \mathrm{MHz}$ and $2698 \mathrm{MHz}$. The interest of bandwidth occupies $1088 \mathrm{MHz}$, which is uniformly divided into 68 subbands encoded as $\left\{B_{1}, B_{2}, \ldots, B_{l}, \ldots, B_{68}\right\}$ with center frequency at $\left\{f_{l}=1602+16 l(\mathrm{MHz})\right\}_{l=1}^{L=68}$. Obviously, the available frequency range for MSS is covered by $\left\{B_{1}, B_{2}, B_{3}, B_{4}, B_{5}, B_{24}\right.$, $\left.B_{25}, B_{66}, B_{67}, B_{68}\right\}$. We take $16 \mathrm{MHz}$ QPSK modulated signal as active PUs to appear in each subband of the set $\left\{B_{1}, B_{2}\right.$, $\left.B_{3}, B_{4}, B_{5}, B_{24}, B_{25}, B_{66}, B_{67}, B_{68}\right\}$ randomly with equal probability. The received signal is corrupted by additive white Gaussian noise (AWGN). From the point of signal detection and spectrum hole search, it is credible that the decision vectors $\widehat{\mathbf{d}}^{\prime(j)}=\left(d_{1}^{(j)}, d_{2}^{(j)}, \ldots, d_{m}^{(j)}, \ldots, d_{M}^{(j)}\right)$ and $\widehat{\mathbf{d}}^{\prime}=$ $\left(\widehat{d}_{1}, \widehat{d}_{2}, \ldots, \widehat{d}_{m}, \ldots, \widehat{d}_{M}\right)$ can be equivalently described as $\widehat{\mathbf{d}}^{\prime(j)}=\left\{d_{l}^{(j)} \mid l \in s, s=[1,2,3,4,5,24,25,66,67,68]\right\}$ and $\widehat{\mathbf{d}}^{\prime}=\left\{\widehat{d}_{l} \mid l \in s\right\}$ which are subset of $\widehat{\mathbf{d}}^{(j)}=\left(d_{1}^{(j)}, d_{2}^{(j)}, \ldots, d_{l}^{(j)}\right.$, $\left.\ldots, d_{L}^{(j)}\right)$ and $\widehat{\mathbf{d}}=\left(\widehat{d}_{1}, \widehat{d}_{2}, \ldots, \widehat{d}_{l}, \ldots, \widehat{d}_{L}\right)$, respectively. We take $N=1024$ samples of the received wideband signal, while the wideband channel experiences frequency-selective fading modeled as a slow Rician fading channel with independent fading gains.

From now on, we aim at analyzing the performance of the abovementioned scheme using the following metrics.

(i) For the spectrum hole detection problem, the key parameters of all spectrum sensing algorithms are detection probability $P_{d}^{(j)}$ and the false alarm probability $P_{f}^{(j)}$ that are evaluated by comparing the local spectrum occupancy decision $\widehat{\mathbf{d}}^{(j)}$ at $j$ th satellite detector with the true state $\mathbf{d}$ over all subbands occupied by Pus; the individual probability of detection and false alarm is defined as follows:

$$
\begin{aligned}
& P_{d}^{(j)}=E\left\{\frac{\mathbf{d}^{T}\left(\mathbf{d}=\widehat{\mathbf{d}}^{(j)}\right)}{\mathbf{1}^{T} \mathbf{d}}\right\}, \\
& P_{f}^{(j)}=E\left\{\frac{(\mathbf{1}-\mathbf{d})^{T}\left(\mathbf{d} \neq \widehat{\mathbf{d}}^{(j)}\right)}{L-\mathbf{1}^{T} \mathbf{d}}\right\},
\end{aligned}
$$

where $E$ denotes expectation, the sets of detection probabilities and false alarm probabilities of $J$ detectors are expressed by $\mathbf{P}_{d}=\left\{P_{d}^{(1)}, P_{d}^{(2)}, \ldots, P_{d}^{(j)}, \ldots, P_{d}^{(J)}\right\}$ and $\mathbf{P}_{f}=$ $\left\{P_{f}^{(1)}, P_{f}^{(2)}, \ldots, P_{f}^{(j)}, \ldots, P_{f}^{(J)}\right\}$. Moreover, when OR fusion rule is employed at the $j$ th satellite detector in the collaborative compressed sensing scheme, the fusion detection probability $Q_{d}$ and fusion false alarm probability $Q_{f}$ can be given by

$$
\begin{aligned}
& Q_{d}=1-\prod_{j=1}^{J}\left(1-P_{d}^{(j)}\right), \\
& Q_{f}=1-\prod_{j=1}^{J}\left(1-P_{f}^{(j)}\right) .
\end{aligned}
$$

When MAJ fusion rule is adopted, $Q_{d}$ and $Q_{f}$ can be evaluated by

$$
\begin{aligned}
& Q_{d}=\sum_{\mathrm{Q}=\left\lfloor J^{\prime} / 2\right\rfloor}^{J^{\prime}} C_{J}^{\mathrm{Q}}\left\{\prod_{j^{\prime}=1}^{\mathrm{Q}} P_{d}^{\left(j^{\prime}\right)}\right\}\left\{\prod_{j^{\prime}=\mathrm{Q}+1}^{J^{\prime}}\left(1-P_{d}^{\left(j^{\prime}\right)}\right)\right\}, \\
& \mathrm{Q}_{f}=\sum_{\mathrm{Q}=\left\lfloor J^{\prime} / 2\right\rfloor}^{J^{\prime}} C_{J}^{\mathrm{Q}}\left\{\prod_{j^{\prime}=1}^{\mathrm{Q}} P_{f}^{\left(j^{\prime}\right)}\right\}\left\{\prod_{j^{\prime}=\mathrm{Q}+1}^{J^{\prime}}\left(1-P_{f}^{\left(j^{\prime}\right)}\right)\right\},
\end{aligned}
$$

where $P_{d}^{\left(j^{\prime}\right)}$ and $P_{f}^{\left(j^{\prime}\right)}$ are chosen from $\mathbf{P}_{d}^{\prime}=$ $\{\underbrace{P_{d}^{\left(1^{\prime}\right)}, P_{d}^{\left(2^{\prime}\right)}, \ldots, P_{d}^{\left(j^{\prime}\right)}, \ldots, P_{d}^{(Q)}}_{\text {Q out of } J^{\prime}}, \ldots, P_{d}^{\left(J^{\prime}\right)}\} \quad$ and $\quad \mathbf{P}_{f}^{\prime}=$ $\{\underbrace{P_{f}^{\left(1^{\prime}\right)}, P_{f}^{\left(2^{\prime}\right)}, \ldots, P_{f}^{\left(j^{\prime}\right)}, \ldots, P_{f}^{(Q)}}, \ldots, P_{f}^{\left(J^{\prime}\right)}\} . \quad$ Obviously, $\quad \mathbf{P}_{d}^{\prime}$ Q out of $J^{\prime}$ 


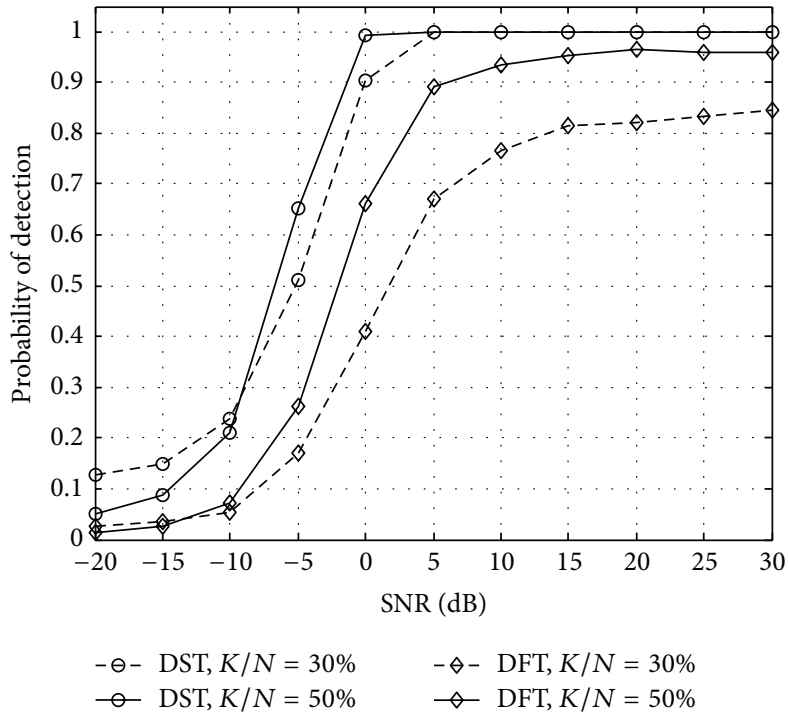

(a)

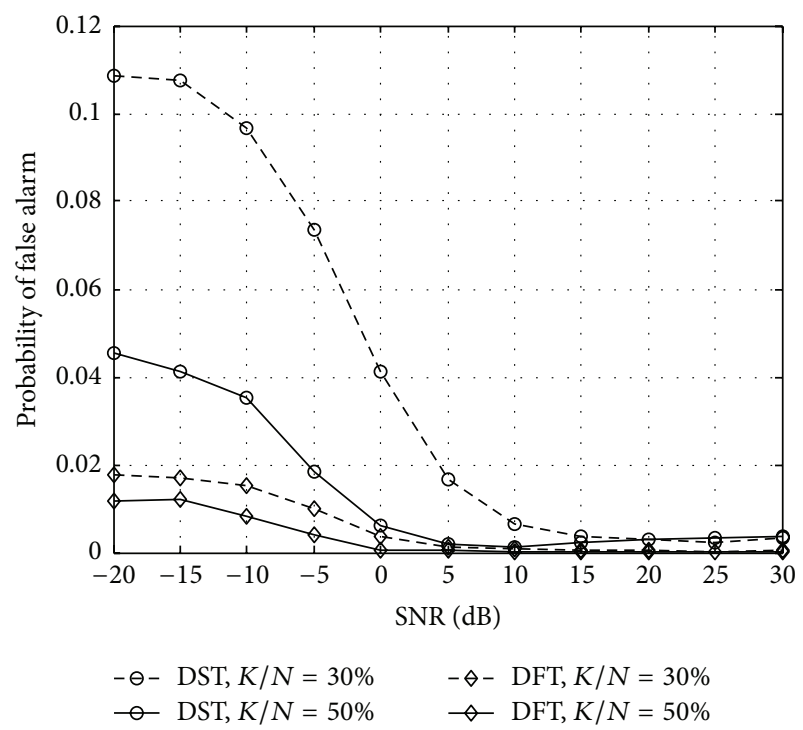

(b)

FIGURE 6: Detection performance versus SNR for different compression rate.

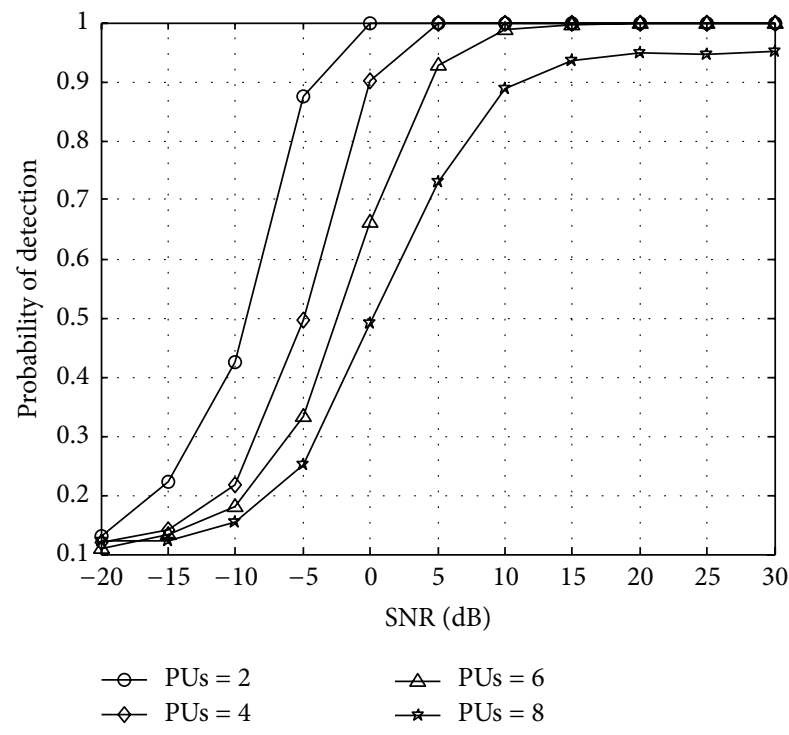

FIGURE 7: Detection probability of different number of active PUs.

and $\mathbf{P}_{f}^{\prime}$ are the realigned form of $\mathbf{P}_{d}$ and $\mathbf{P}_{f}$, and $\mathbf{P}_{d}^{\prime}=\mathbf{P}_{d}$, $\mathbf{P}_{f}^{\prime}=\mathbf{P}_{f}$, and $J^{\prime}=J$.

(ii) To evaluate the performance of spectrum reconstruction accuracy, we use normalized mean squared error (NMSE) of the reconstructed spectrum $\widehat{r}_{f}$, which is defined as

$$
\mathrm{NMSE}=E\left\{\frac{\left\|\hat{r}_{f}-r_{f}\right\|_{2}^{2}}{\left\|r_{f}\right\|_{2}^{2}}\right\} .
$$

5.2. Local Compressed Sensing Performance Based on DFT and DST. In this section, we compare the detection performance

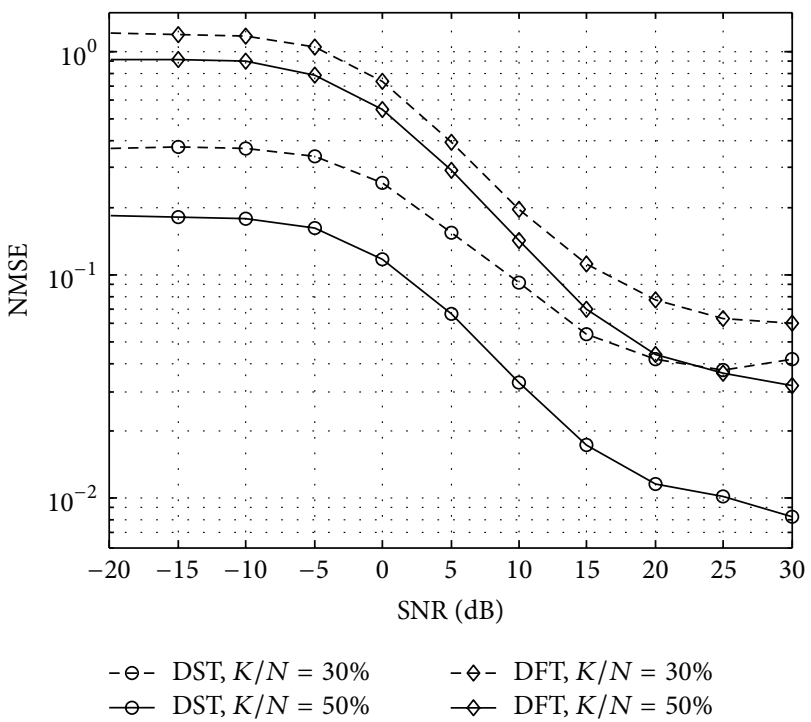

FIGURE 8: Recovery performance NMSE for different compressive sensing schemes.

of the proposed DST-WSCS scheme with that of conventional DFT-WSCS scheme in terms of detection probability, false alarm probability, reconstruction accuracy, and running time. The Monte-Carlo simulation method of 1000 times is used to evaluate the performance of single detector.

(i) $\mathbf{P}_{d}$ and $\mathbf{P}_{f}$ versus SNR, Compression Ratio, and the Number of Active PUs. Figure 6 depicts detection probability and false alarm probability versus SNR under different values of compression ratio when four subbands are randomly occupied. It indicates that detection probability improves and false alarm probability decreases obviously with SNR or compressive 


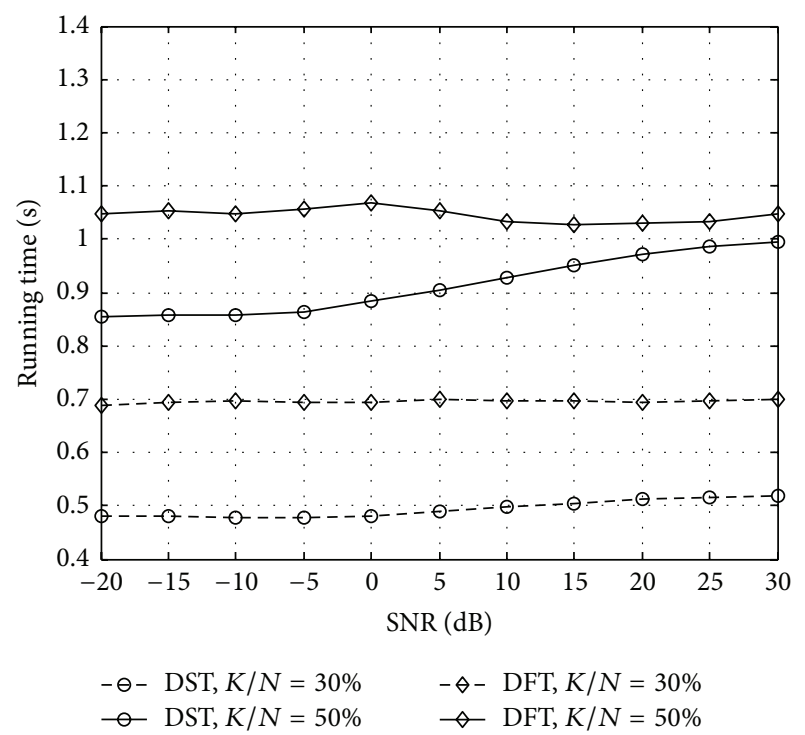

FIGURE 9: Running time of individual satellite detector for different compressive sensing schemes.

rate increasing. More measurements means higher sampling burden and energy consumption, and a higher SNR can afford less compression ratio during data acquisition. The proposed DST-WSCS provides higher successful detection than that of the conventional method DFT-WSCS, although it heightens the false alarm probability to a certain extent in low SNR. On the other hand, we could reduce the false alarm probability through heightening threshold factor, as depicted in Figure 10.

Figure 7 shows the relationship between the detection performance and the number of active PUs based on DST method when $K / N=30 \%$. It is equivalent to comparing performances for different degree of sparsity of the wideband spectrum. It can be concluded that detection performance improves as degree of sparsity of the wideband spectrum increases. This is because the theory of compressed sensing is based on the sparsity of spectrum. Additionally, the number of active primary signals has little impact on the false alarm probability.

(ii) Sparse Spectrum Recovery Performance of NMSE versus $S N R$ and Compression Ratio. Figure 8 depicts the NMSE of the estimated spectrum for various values of compression ratio and SNR, when there are four active PUs. From Figure 8, we can see DST-WSCS outperforms DFT-WSCS. And higher SNR or compression rate means higher reconstruction accuracy.

(iii) Running Time for Individual Satellite Detector versus SNR and Compression Ratio. Figure 9 gives the execution time of the proposed detection algorithm for different compression ratio $K / N$, with four active PUs. It shows that the detection time is closely related to the compressive ratio, while it has little connection with SNR. The average detection time of DST-based compressive sensing is smaller than that of the DFT-based by at least $20 \%$, due to the real value of DST operation. Summarily, the DST-based method costs less processing time, as well as lower processing complexity than DFT-based method, which is of great significance in MSS for the limitation of processing capability and time-varying spectrum.

(iv) The Effect of Threshold Factor on Detecting and ROC Performance. Figure 10 focuses on the effect of threshold factor on detection performance and the receiver operating characteristic (ROC) performance, when $K / N$ is $50 \%$ and SNR is $0 \mathrm{~dB}$. It indicates that either detection or false alarm detection degrades as threshold factor increases. Obviously, the proposed scheme of DST-WSCS performs better than DFT-WSCS in detection probability for the same false alarm rate. It should be pointed out that we take 1.4 as threshold factor through the full paper for the sake of the balance between detection and false alarm probability, which also explains that the false alarm probability of DST-WSCS is a little higher than that of DFT-WSCS, as illustrated in Figure 6.

\subsection{Cooperative Compressed Sensing Performance Based on DFT and DST}

(i) The Effect of Different Number of Cooperators on $Q_{d}$ and $Q_{f}$. Figure 11 compares the cooperative compressed sensing performance based on DFT and DST for different cooperators with OR fusion rule, when there are four subbands randomly occupied and compression rate $K / N$ is $50 \%$. It is clear that the probability of detection is enhanced as the number of cooperators increases. This is because collaborative scheme brings spatial diversity gain. However, the false alarm probability becomes worse, especially for low SNR; this is according to the theoretical analysis mentioned above. Fortunately, the absolute value of $Q_{f}$ is extremely small and would not bring serious interference to PUs. In order to reduce $Q_{f}$ with accepted $Q_{d}$ that provides sufficient protection to PUs, we can alternatively heighten threshold factor as shown in Figure 10 or employ MAJ fusion rule as demonstrated in Figure 12.

(ii) The Effect of Decision Fusion Rule on $Q_{d}$ and $Q_{f}$. Figure 12 focuses on the effect of decision fusion rule on cooperative compressed sensing performance based on DFT and DST with three cooperators, when there are four subbands randomly occupied and compression rate $K / N$ is $50 \%$. It is obvious that the utilization of MAJ fusion rule can reduce both the probability of detection and the false alarm probability greatly. Nevertheless, the DST method performs better for the robustness to noise in the respect of detection probability. We suggest that the OR fusion rule and MAJ fusion rule be employed jointly in practice, which can provide higher probability of detection and lower false alarm probability, respectively. 


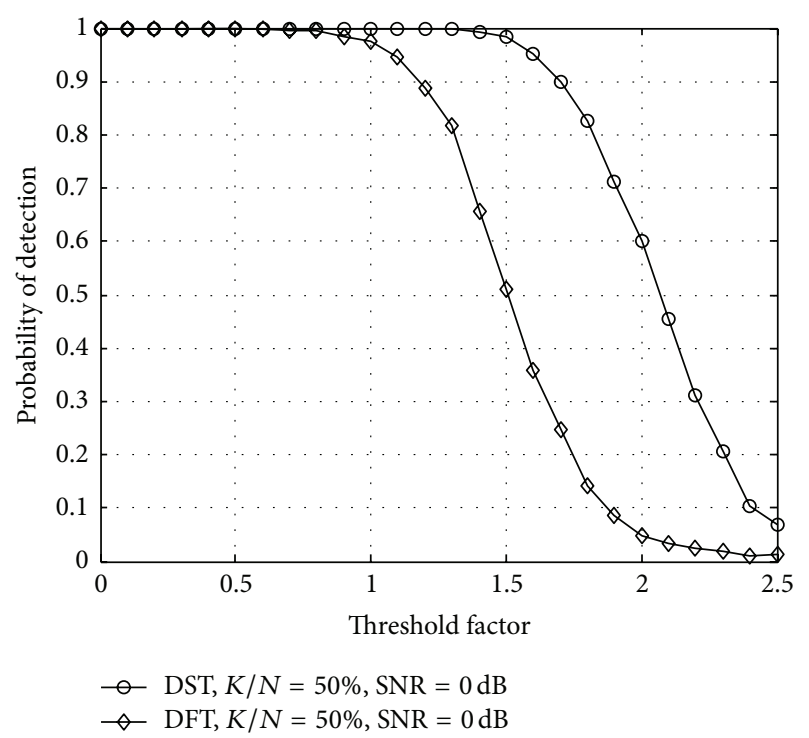

(a)

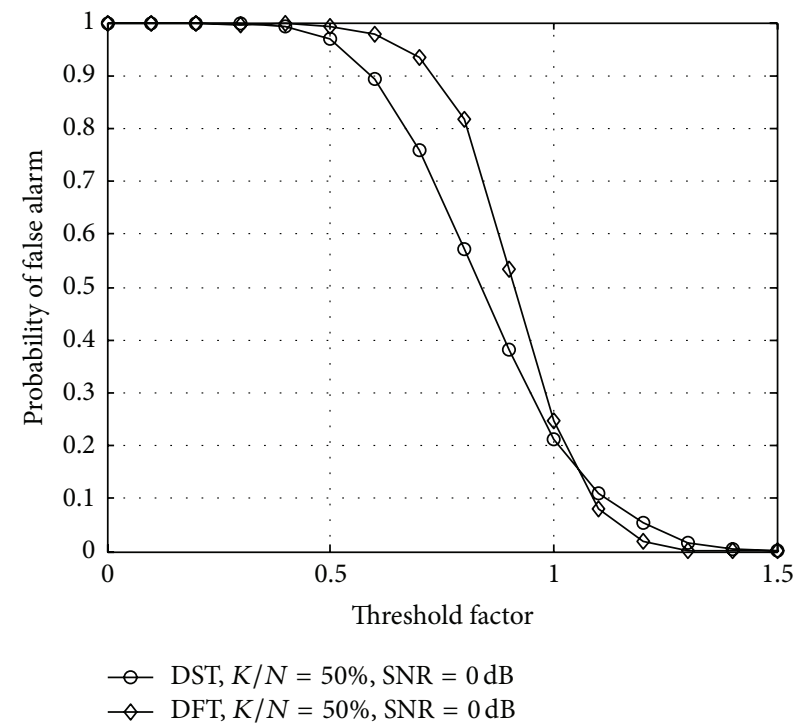

(b)

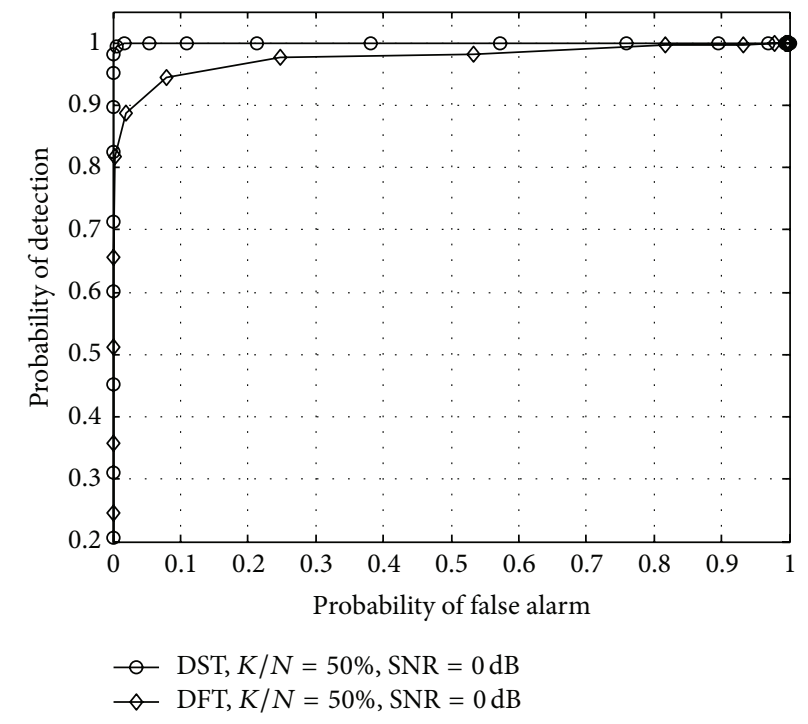

(c)

FIGURE 10: The performances of detecting and ROC versus threshold factor.

\section{Conclusion}

In this paper, we proposed a novel wideband spectrum compressed sensing scheme based on DST to resolve the frequency resource scarcity problem in mobile satellite systems. Furthermore, we also presented collaborative compressed sensing scheme to mitigate the adverse effect of fading on sensing performance. Additionally, given consideration to balance between detection probability and false alarm probability, we suggested the joint fusion rule based on the OR and MAJ fusion rule. Simulation results show that notable improvement in the detection probability, processing time, and reconstruction accuracy can be obtained using DSTbased sensing algorithm compared with conventional DFTbased compressive sensing, and spatial diversity gain can be achieved via cooperative sensing at the expense of little loss in false alarm probability, which can be compromised by either heightening threshold factor or the jointed decision fusion rule. More applicable decision fusion rule for collaboration and universal reconstruction algorithm will be our future direction of research.

\section{Competing Interests}

The authors declare that there is no conflict of interests regarding the publication of this paper.

\section{Acknowledgments}

This work is supported by National Natural Science Foundation of China (61571464, 91338201, and 91438109) 


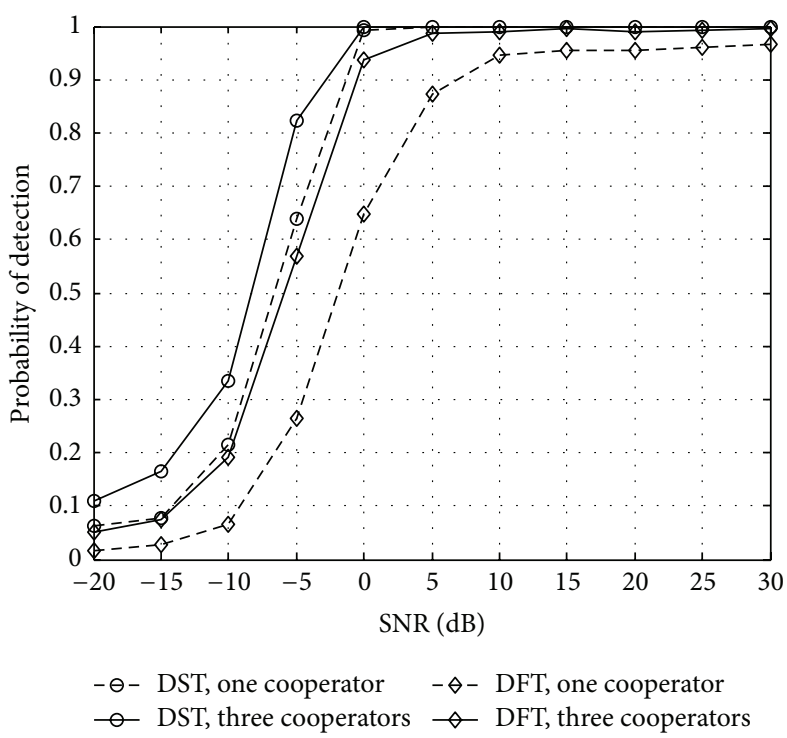

(a)

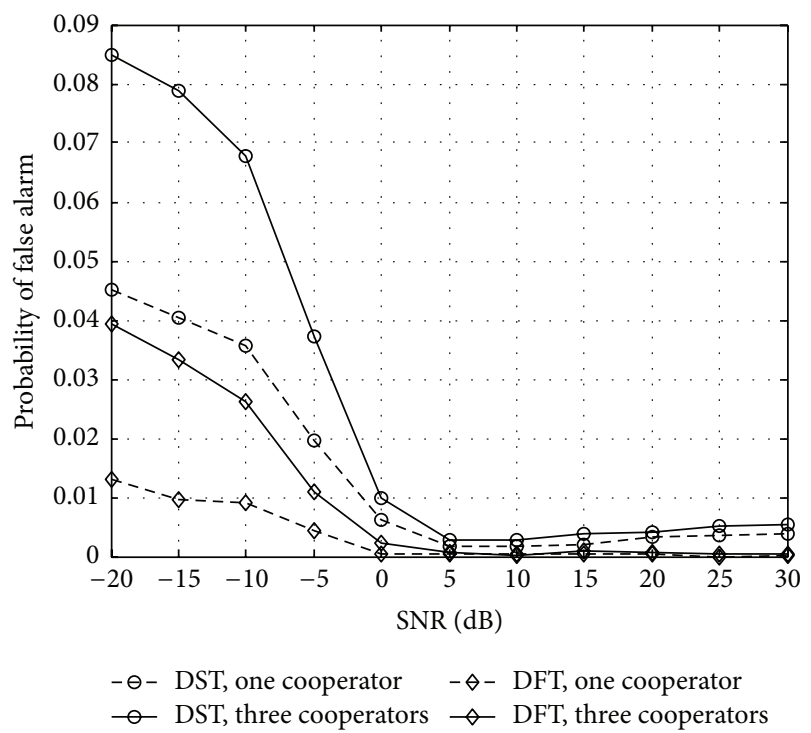

(b)

FIGURE 11: Cooperative compressed sensing performance versus the number of cooperators.

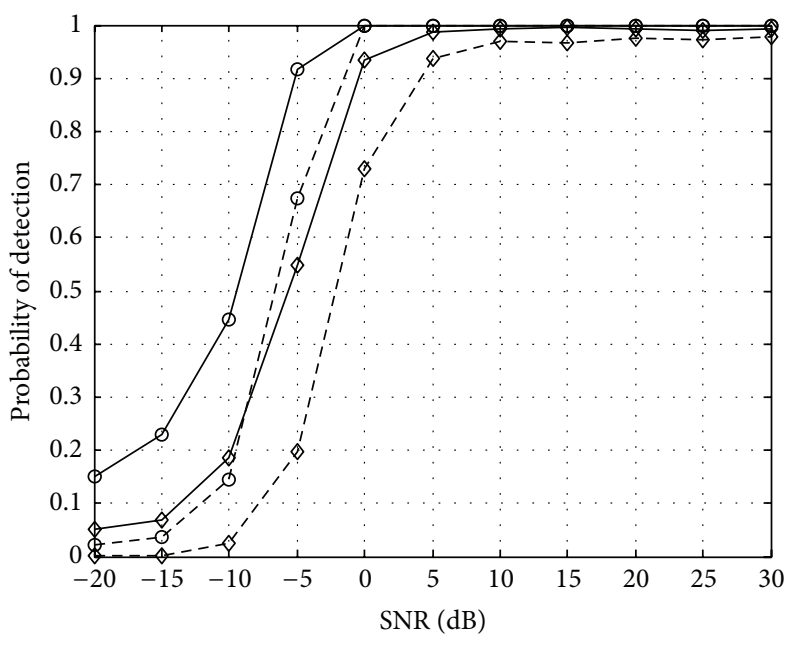

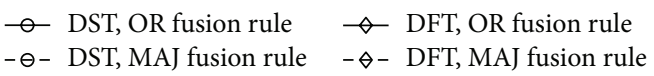

(a)

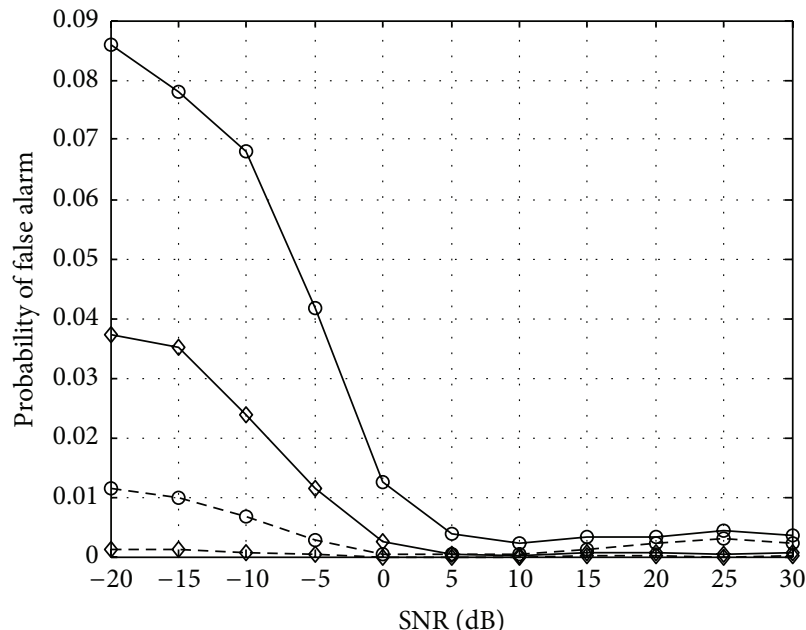

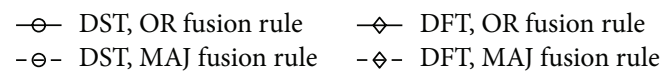

(b)

FIGURE 12: Detection performance comparison at different decision fusion rules.

and National High Technology Research and Development Program of China ("863" Program) (2012AA121605, 2012AA01A503, and 2012AA01A510).

\section{References}

[1] F. Alagöz and G. Gür, "Energy efficiency and satellite networking: a holistic overview," Proceedings of the IEEE, vol. 99, no. 11, pp. 1954-1979, 2011.

[2] P. Chini, G. Giambene, and S. Kota, "A survey on mobile satellite systems," International Journal of Satellite Communications and Networking, vol. 28, no. 1, pp. 29-57, 2010.
[3] N. Celandroni, E. Ferro, A. Gotta et al., "A survey of architectures and scenarios in satellite-based wireless sensor networks: system design aspects," International Journal of Satellite Communications and Networking, vol. 31, no. 1, pp. 1-38, 2013.

[4] A. K. Maini and V. Agrawal, Satellite Technology. Principles and Applications, John Wiley \& Sons, Hoboken, NJ, USA, 2011.

[5] P. Muri and J. McNair, "A survey of communication sub-systems for intersatellite linked systems and cubesat missions," Journal of Communications, vol. 7, no. 4, pp. 290-308, 2012.

[6] J. Albuquerque, "Key note speech-what is going on in commercial satellite communications," in Proceedings of the Keynote of the International Workshop on Satellite and Space Communications (IWSSC '07), Salzburg, Austria, September 2007. 
[7] A. Arcidiacono, D. Finocchiaro, and S. Grazzini, "Broadband mobile satellite services: the Ku-band revolution," in Proceedings of the Tyrrhenian International Workshop on Digital Communications (TIWDC '06), Island of Ponza, Italy, September 2006.

[8] Y. Wang and G. Zhang, "Compressed wideband spectrum sensing based on discrete cosine transform," The Scientific World Journal, vol. 2014, Article ID 464895, 5 pages, 2014.

[9] S. Haykin, "Cognitive radio: brain-empowered wireless communications," IEEE Journal on Selected Areas in Communications, vol. 23, no. 2, pp. 201-220, 2005.

[10] D. L. Donoho, “Compressed sensing," IEEE Transactions on Information Theory, vol. 52, no. 4, pp. 1289-1306, 2006.

[11] J.-A. Bazerque and G. B. Giannakis, "Distributed spectrum sensing for cognitive radio networks by exploiting sparsity," IEEE Transactions on Signal Processing, vol. 58, no. 3, pp. 18471862, 2010.

[12] Z. Tian and G. B. Giannakis, "Compressed sensing for wideband cognitive radios," in Proceedings of the IEEE International Conference on Acoustics, Speech and Signal Processing (ICASSP '07), pp. IV-1357-IV-1360, Honolulu, Hawaii, USA, April 2007.

[13] M. F. Duarte and Y. C. Eldar, "Structured compressed sensing: from theory to applications," IEEE Transactions on Signal Processing, vol. 59, no. 9, pp. 4053-4085, 2011.

[14] G. Leus and D. D. Ariananda, "Power spectrum blind sampling," IEEE Signal Processing Letters, vol. 18, no. 8, pp. 443-446, 2011.

[15] Z. Tian, Y. Tafesse, and B. M. Sadler, "Cyclic feature detection with sub-nyquist sampling for wideband spectrum sensing," IEEE Journal on Selected Topics in Signal Processing, vol. 6, no. 1, pp. 58-69, 2012.

[16] G. Peyre, "Best basis compressed sensing," IEEE Transactions on Signal Processing, vol. 58, no. 5, pp. 2613-2622, 2010.

[17] L. R. Amado, E. S. C. Losqui, F. P. V. de Campos, A. A. M. de Medeiros, and M. V. Ribeiro, "Spectrum sensing for powering power line communications," in Simposio Brasileiro de Telecomunicacoes, Brasília, Brazil, September 2012.

[18] F. Zeng, C. Li, and Z. Tian, "Distributed compressive spectrum sensing in cooperative multihop cognitive networks," IEEE Journal on Selected Topics in Signal Processing, vol. 5, no. 1, pp. 37-48, 2011.

[19] Z. Li, F. R. Yu, and M. Huang, "A distributed consensus-based cooperative spectrum-sensing scheme in cognitive radios," IEEE Transactions on Vehicular Technology, vol. 59, no. 1, pp. 383-393, 2010.

[20] J. Meng, W. Yin, H. Li, E. Hossain, and Z. Han, "Collaborative spectrum sensing from sparse observations in cognitive radio networks," IEEE Journal on Selected Areas in Communications, vol. 29, no. 2, pp. 327-337, 2011.

[21] M. Jia, X. Liu, X. Gu, and Q. Guo, "Joint cooperative spectrum sensing and channel selection optimization for satellite communication systems based on cognitive radio," International Journal of Satellite Communications and Networking, 2015.

[22] Y. Wang, G. Zhang, D. Bian, L. Gou, and W. Zhang, "Collaborative wideband compressed signal detection in interplanetary internet," Frequenz, vol. 68, no. 7-8, pp. 389-401, 2014.

[23] Sparse Lab Toolbox, https://sparselab.stanford.edu/. 


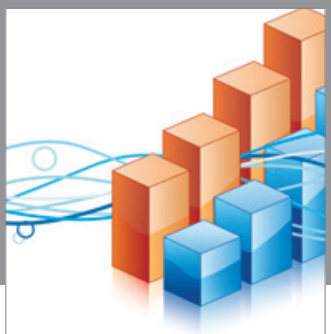

Advances in

Operations Research

vatem alat4

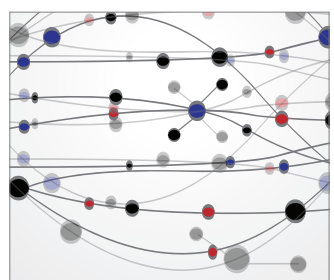

\section{The Scientific} World Journal
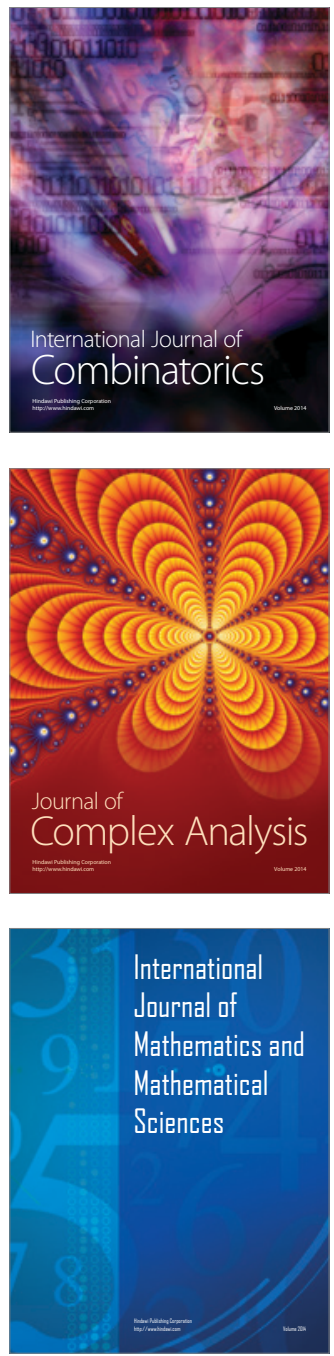
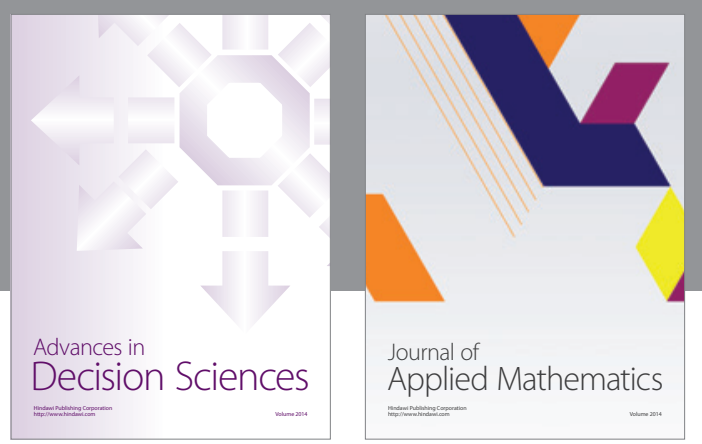

Algebra

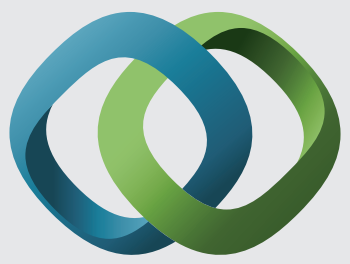

\section{Hindawi}

Submit your manuscripts at

http://www.hindawi.com
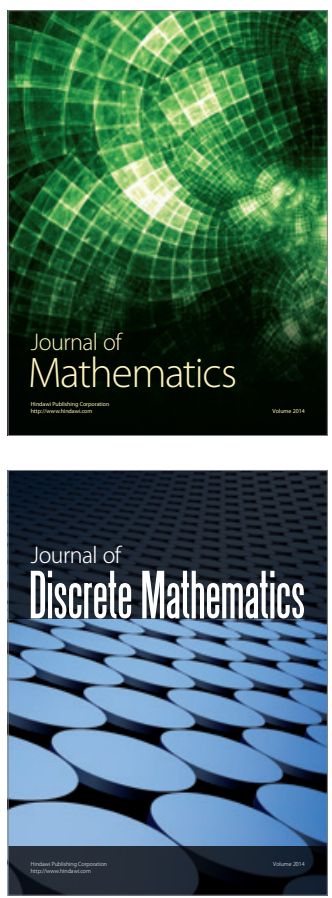

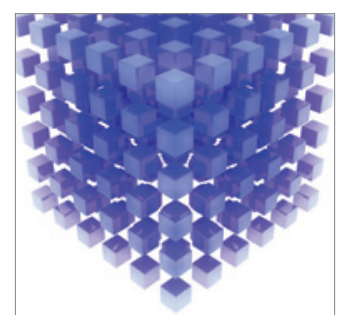

Mathematical Problems in Engineering
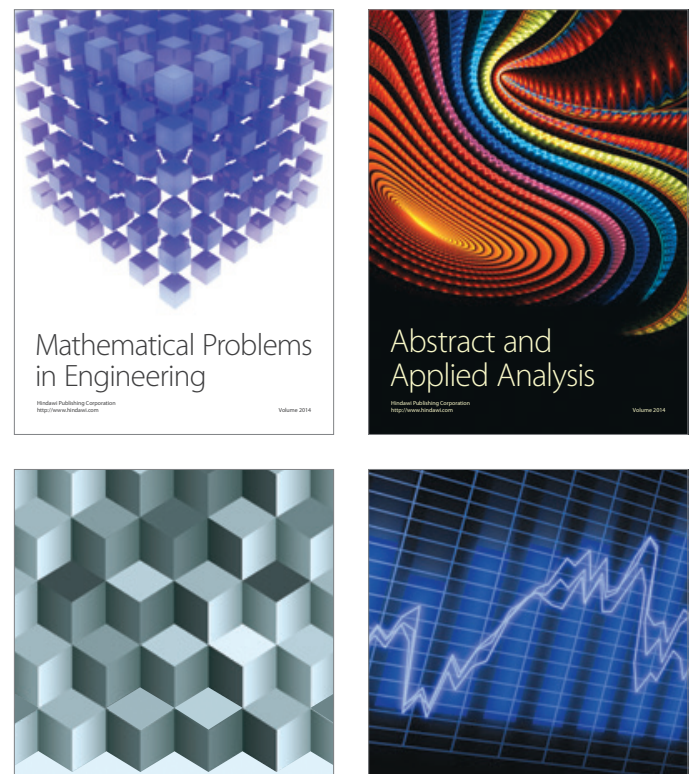

Journal of

Function Spaces

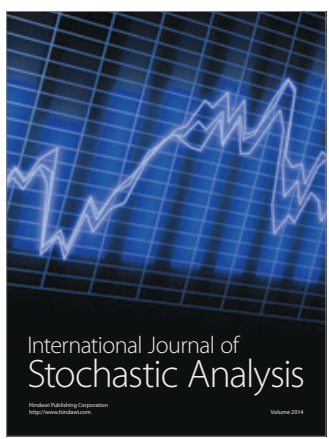

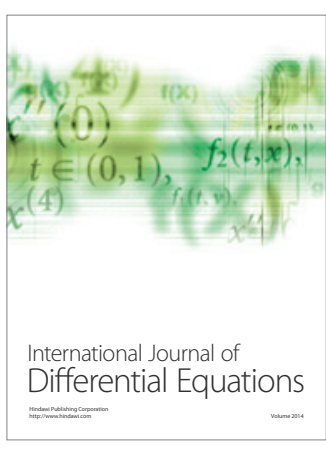
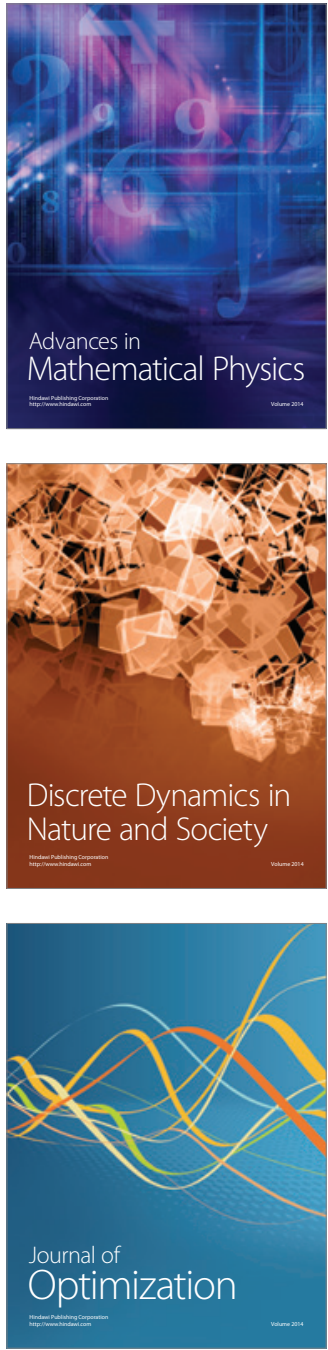